\title{
Anlegen eines Augenverbands - Schritt für Schritt
}

Stefan J Lang, Cornelius Wiedenmann, Philip Keye, Thomas Reinhard

Die Notwendigkeit von Augenverbänden in verschiedenen Situationen wird kontrovers diskutiert. Trotzdem zählt das Anlegen eines Augenverbands zu den täglich notwendigen Handgriffen in der augenärztlichen Tätigkeit. Im Folgenden präsentieren wir das sichere und effektive Anlegen eines Augenverbands.

Grundsätzlich gibt es verschiedene Verbandsformen, welche unterschiedliche Anwendungsgebiete haben. Ein Augensalbenverband wird bei oberflächlichen Verletzungen oder einer Hornhauterosio eingesetzt. Verbände mit Schutzkapseln dienen dem postoperativen Schutz des Auges. Uhrglasverbände werden bei Lagophthalmus eingesetzt. Druckverbände werden nach Lidoperationen angewandt. Sowohl beim Anlegen als auch beim Entfernen eines Augenverbands müssen alle Basis-Hygienemaßnahmen berücksichtigt werden.

\section{Schritt 1 Hygienische} Händedesinfektion ( $\bullet$ Abb. 1)

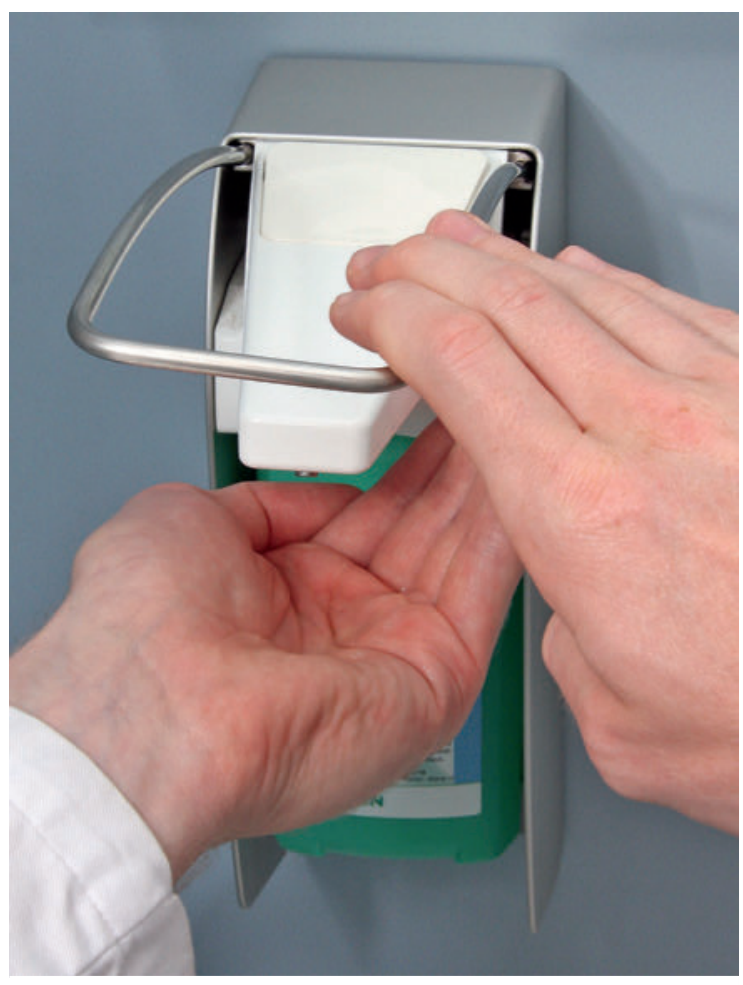

Abb. 1
Insofern die Verbandsapplikation nicht unter sterilen Bedingungen im Operationssaal geschieht, sollte zunächst eine hygienische Händedesinfektion erfolgen. Erst nachdem das Desinfektionsmittel getrocknet ist, kann der nächste Schritt durchgeführt werden.

\section{Schritt 2 Korrekte Sitzposition ( $\mathbf{A b b}$. 2)}

Sowohl beim Anlegen als auch bei der Abnahme des Verbands sollte der Patient entweder mit dem Kopf angelehnt sitzen oder sich in einer liegenden Position befinden.

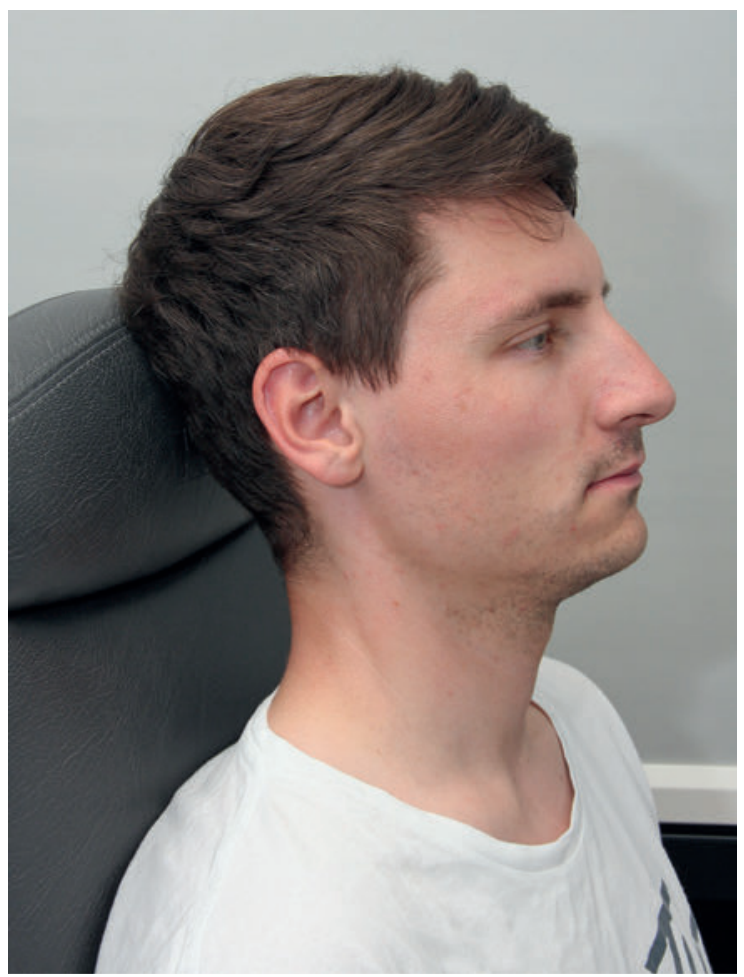

Abb. 2 
Schritt 3 Applikation von Augentropfen oder Augensalben ( $\mathbf{A} \mathbf{b b} \mathbf{3}$ )

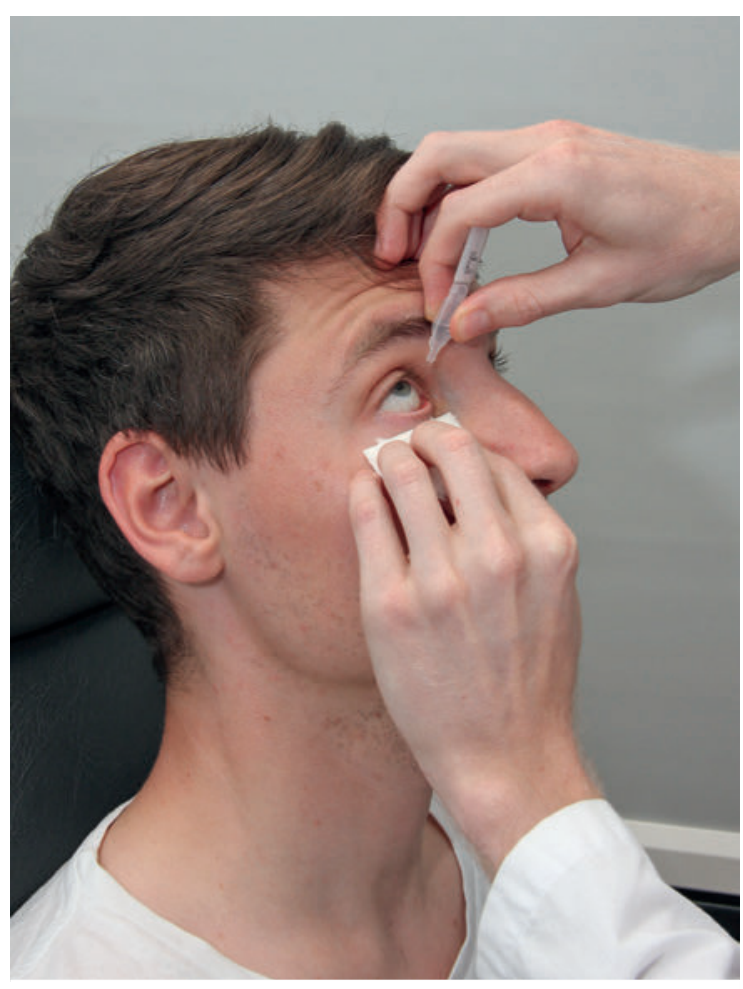

-Abb. 3

Je nach Verbandsform werden Augentropfen oder Augensalben verabreicht. Der Patient blickt hierzu nach oben. Das Unterlid wird ektropioniert und die Applikation erfolgt im temporal unteren Fornix.
Schritt 4 Anlegen des Verbands Augensalbenverband ( $\boldsymbol{A}$ Abb.4)

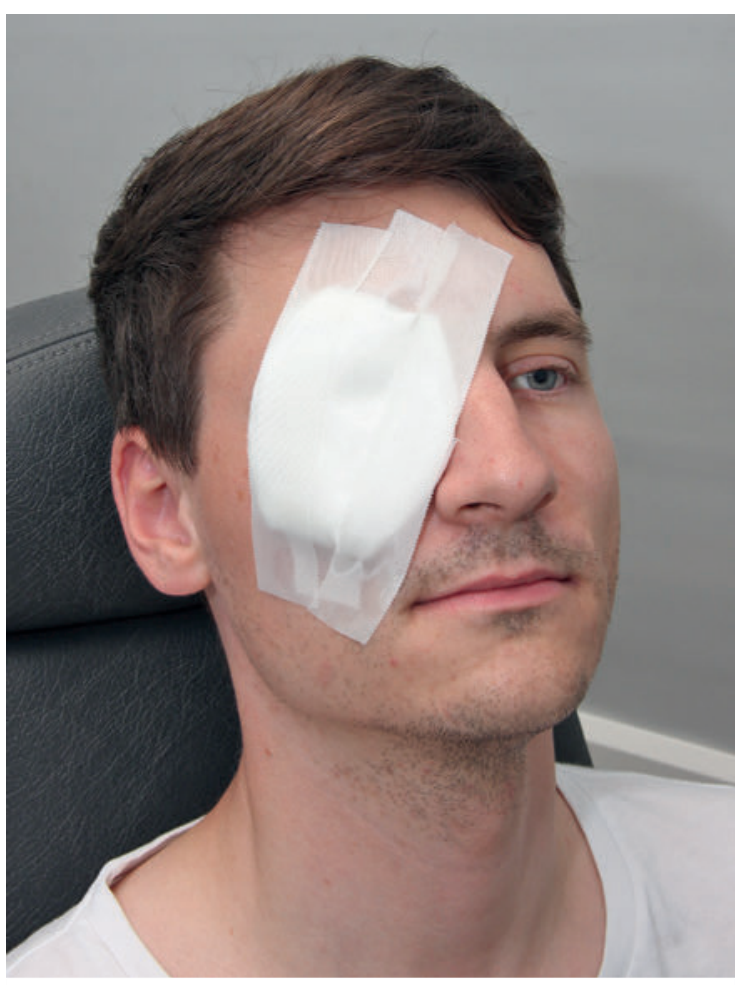

- Abb. 4

Eine sterile Augenkompresse wird mit 2-3 hautfreundlichen Pflasterstreifen fixiert. Der Patient sollte das Auge unter dem Verband geschlossen lassen. 


\section{Druckverband ( $\bullet$ Abb.5)}

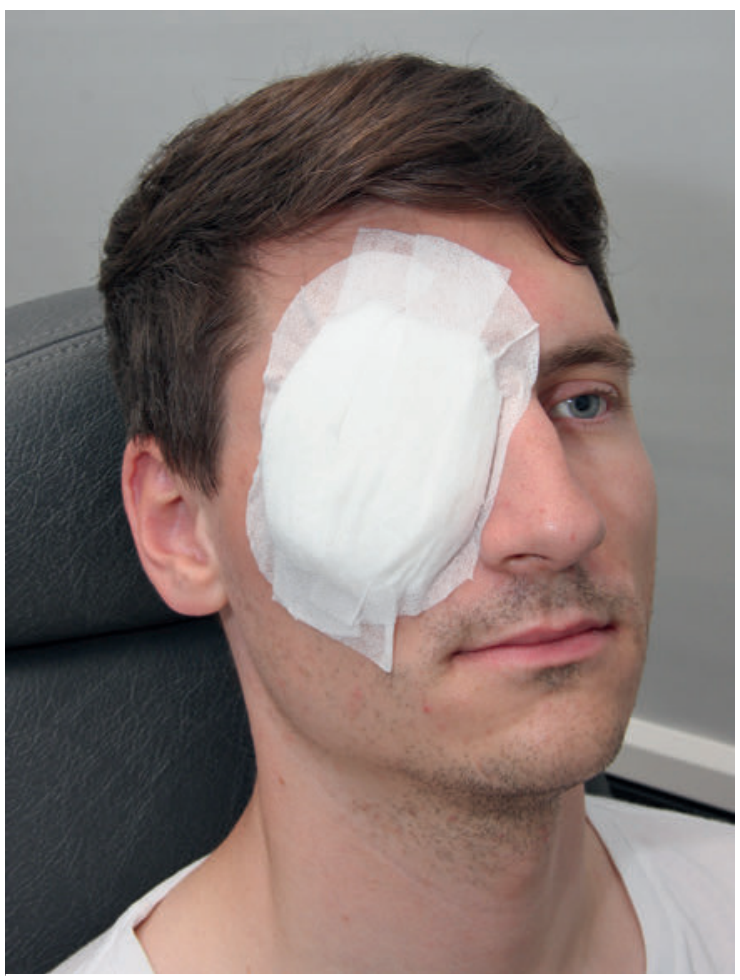

Abb. 5

Es werden zunächst ein bis zwei befeuchtete sterile Kugeltupfer und/oder eine halbiert übereinandergelegte sterile Kompresse aufgelegt. Darüber werden 1-2 sterile trockene Kompressen gelegt. Der Verband wird dann mit 3 breiten Pflasterstreifen fest fixiert.

\section{Schutzkapsel}

Die Schutzkapsel wird mit 2-3 hautfreundlichen Pflasterstreifen befestigt.

\section{Uhrglasverband ( $\bullet$ Abb. $\mathbf{6})$}

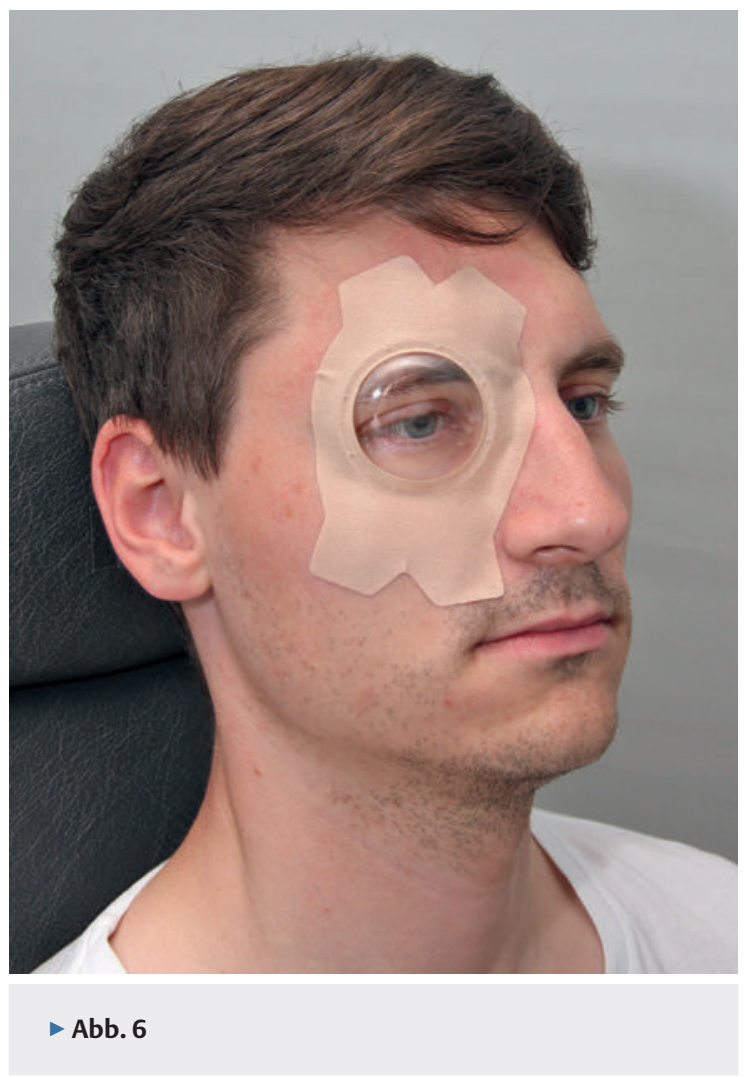

Der Uhrglasverband wird mit der selbstklebenden Fläche so angebracht, dass diese dicht abschließt.

\section{Schritt 5 Abnehmen des Verbands}

Der anliegende Augenverband wird vorsichtig entfernt und das Verbandsmaterial sofort entsorgt. Gegebenenfalls wird eine kurze Reinigung mit einem feuchten sterilen Tupfer durchgeführt. 
Die Autorinnen/Autoren geben an, dass kein Interessenkonflikt besteht.

\section{Autorinnen/Autoren}

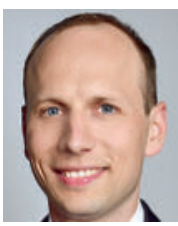

\section{Stefan Lang}

PD Dr. med., 2003-2009 Medizinstudium in Ulm. 2011 Promotion. 2010-2015 Facharztausbildung an der Universitätsklinik Freiburg. 2015 Facharzt für Augenheilkunde. 2015 Fellow of the European Board of Ophthalmology (FEBO). Seit 2015 Funktionsoberarzt an der Klinik für Augenheilkunde der Universitätsklinik Freiburg.

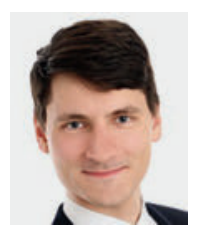

\section{Cornelius Wiedenmann}

Dr. med., 2011-2018 Medizinstudium in Freiburg i. Br. 2019 Promotion. Seit 2018 Assistenzarzt an der Klinik für Augenheilkunde der Universitätsklinik Freiburg.

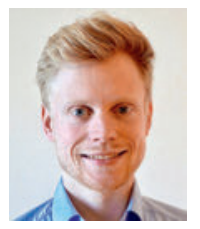

\section{Philip Keye}

2011-2018 Medizinstudium in Freiburg i. Br., seit 2019 Assistenzarzt an der Klinik für Augenheilkunde der Universitätsklinik Freiburg.

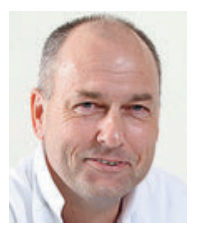

\section{Thomas Reinhard}

Prof. Dr. med., seit 2003 Ärztlicher Direktor der Universitäts-Augenklinik Freiburg. Seit 2014 Generalsekretär der Deutschen Ophthalmologischen Gesellschaft (DOG). Neben zahlreichen weiteren Aufgaben ist er Mitglied des Wissenschaftlichen Beirats der Bundesärztekammer. Seine Schwerpunkte umfassen Hornhauteingriffe, außerdem Kataraktoperationen und Glaukomoperationen; er hat über 25000 Eingriffe durchgeführt.

\section{Korrespondenzadresse}

PD Dr. Stefan J. Lang, FEBO

Universitätsklinikum Freiburg

Klinik für Augenheilkunde

Killianstr. 5

79106 Freiburg

stefan.lang@uniklinik-freiburg.de

\section{Bibliografie}

DOI https://doi.org/10.1055/a-0992-0047

Augenheilkunde up2date 2020; 10: 116-119

(c) Georg Thieme Verlag KG Stuttgart · New York

ISSN 0023-2165

\section{Service für unsere Leser}

\section{Schon registriert?}

Bei Thieme CME Punkte online einreichen und verwalten

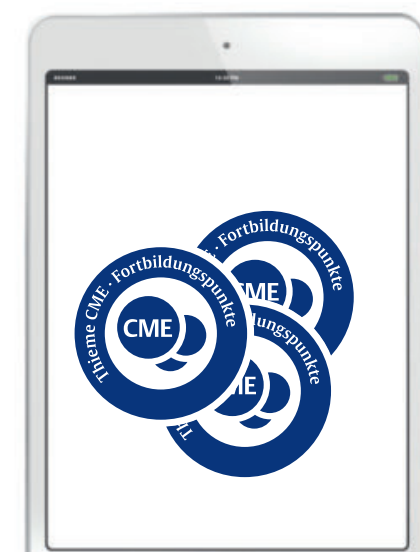

Gleich anmelden:

www.cme.thieme.de

up2date - Fortbildung mit dem roten Faden 